\section{Reflexão sobre a imigração japonesa no Brasil sob o ângulo da adiposidade corporal}

\author{
Reflection about Japanese immigration to Brazil \\ under the light of body adiposity
}

Sandra R. G. Ferreira', Bianca de Almeida-Pittito',

Japanese-Brazilian Diabetes Study Group (JBDS Group) ${ }^{2}$

\section{RESUMO}

Populações migrantes representam oportunidade para se investigar a contribuição de fatores ambientais na gênese da obesidade e suas comorbidades. Em 1993, o Japanese-Brazilian Diabetes Study Group estudou a prevalência de diabetes e doenças associadas em nipobrasileiros residentes em Bauru, SP. Utilizando critérios específicos para asiáticos, 22,4\% dos nipo-brasileiros foram caracterizados como portadores de excesso de peso nessa primeira fase do estudo. Na segunda fase, em 2000 , essa prevalência subiu para $44,2 \%$, e $50,3 \%$ apresentavam obesidade central. Essa população também apresentava alta prevalência de diabetes tipo 2, hipertensão e dislipidemia, componentes da síndrome metabólica. $0 \mathrm{JBDS}$ Group também mostrou a associação entre hábitos ocidentais, especialmente alimentação rica em gordura saturada, e a ocorrência de síndrome metabólica. Em 2005, motivado por esses achados, o JBDS Group iniciou a terceira fase do estudo que constou de programa de intervenção com base em orientação para dieta saudável e prática de atividade física, utilizando recursos factíveis em termos de saúde pública no Brasil. Após um ano de intervenção, o JBDS Group observou diminuição nos parâmetros antropométricos, pressão arterial e níveis de glicemia e colesterol. Tempo de acompanhamento maior é necessário para avaliar a persistência desses benefícios e o impacto deles no risco de desenvolver diabetes e eventos cardiovasculares. Arq Bras Endocrinol Metab. 2009;53(2):175-182.

\section{Descritores}

Adiposidade corporal; obesidade abdominal; imigrantes japoneses; síndrome metabólica

\begin{abstract}
Migrant populations represent a good opportunity to investigate the role of environmental factors for the genesis of obesity and its comorbidities. The Japanese-Brazilian Diabetes Study Group studied the prevalence of diabetes and related disorders in Japanese-Brazilians from Bauru, SP, in 1993. Using specific criteria for Asian, 22.4\% of the Japanese-Brazilians were found as having overweight in this first phase of the study. In the second phase, in 2000 , this prevalence increased to $44.2 \%$ and $50.3 \%$ had central obesity. This population also had high prevalence of type 2 diabetes, hypertension and dyslipidemia, components of the metabolic syndrome. The JBDS Group also showed the association between Occidentalized habits, mainly a rich saturated-fat-diet, and the occurrence of the metabolic syndrome. In 2005, motivated by these findings, the JBDS Group started the third phase of the study which was an intervention program based on healthy diet and physical activity recommendations, using resources that could be feasible in terms of public health in Brazil. After one year-intervention program, the JBDS Group observed a decrease in anthropometric parameters, blood pressure and plasma glucose and cholesterol levels. It will be necessary a longer follow-up to evaluate the maintainance of these benefits and their impact in the risk development of diabetes and cardiovascular events. Arq Bras Endocrinol Metab. 2009;53(2):175-182.
\end{abstract}

'Departamento de Nutrição, Faculdade de Saúde Pública da Universidade de São Paulo (FSP/USP), São Paulo, SP, Brasil ${ }^{2}$ Membros do Japanese-Brazilian Diabetes Study Group (JBDSG): Amélia T. Hirai, Helena Harima, Katsumi Osiro, Mario Kikuchi, Renata Damião, Suely G. A. Gimeno (Departamento de Medicina Preventiva, Universidade Federal de São Paulo (Unifesp), São Paulo, SP, Brasil); Daniela S. Sartorelli, Laércio J. Franco (Departamento de Medicina Social, Faculdade de Medicina de Ribeirão Preto da Universidade de São Paulo (FMRPUSP), Ribeirão Preto, SP, Brasil); Katsunori Wakisaka (JapaneseBrazilian Study Center, São Paulo, $\mathrm{SP}$, Brasil); Luiza K. Matsumura, Regina C. S. Moisés (Disciplina de Endocrinologia, Departamento de Medicina, Unifesp, São Paulo, SP, Brasill; Marly A. Cardoso, Sandra R.G. Ferreira (Departamento de Nutrição, FSP/USP, São Paulo, SP, Brasil); Nilce Tomita (Faculdade de Odontologia de Bauru, Universidade de São Paulo (USP), Bauru, SP, Brasil); Rita Chaim (Faculdade de Nutrição, Universidade Sagrado Coração de Jesus (USC), Bauru, SP, Brasil)

Correspondência para: Sandra R. G. Ferreira Departamento de Nutrição da Faculdade de Saúde Pública, USP Av. Dr. Arnaldo, 715, $2^{\circ}$ andar 01246-904 - São Paulo, SP, Brasil sandrafv@usp.br

Recebido em 19/Dez/2008 Aceito em 03/Fev/2009 


\section{INTRODUÇÃO}

$\mathrm{P}$ ossivelmente, a preocupação com o meio ambiente nunca esteve tão viva como na atualidade. A afirmação soa verdadeira sob os mais variados ângulos do conhecimento científico. Ao mesmo tempo que a saúde ambiental vem sendo seriamente afetada por comportamentos do homem moderno, facilidades no meio ambiente, inventadas pelo homem, têm paradoxalmente revertido em certos malefícios à sua saúde. Propõe-se aqui focalizar o impacto de fatores ambientais sobre a saúde humana e, no que diz respeito a ela, como estes contribuem para acumular gordura corporal a ponto de resultar obesidade. Essa consequência das mudanças ocorridas no ambiente (fartura de alimentos, processamento de alimentos, avanços tecnológicos e inatividade física) participa na gênese das principais doenças crônicas que matam o homem. Vários modelos interessantes se prestam para investigar a participação de fatores extrínsecos na gênese da obesidade. As lições dos experimentos animais são muito úteis para se estabelecer relações causais e conhecer mecanismos fisiopatológicos. Importantes hipóteses testadas nesses estudos tiveram sua origem em observações relatadas em estudos epidemiológicos. Particularmente, estudos de populações que migram para ambiente com características geográficas e culturais distintas representam oportunidade ímpar para se investigar o papel do fator ambiental na gênese de doenças.

A literatura tem exemplos clássicos de estudos de populações migrantes apontando o impacto do novo ambiente no aumento da prevalência de obesidade.

Talvez o mais importante, ou, pelo menos, o mais famoso estudo nesta linha, é o dos índios Pima (1), descendentes dos Hohokans que habitaram a região da Piméria, antigamente em terras mexicanas, hoje estado do Arizona, Estados Unidos. Esta população apresentava índice de massa corporal (IMC) normal quando trabalhava arduamente na zona rural, onde desenvolveram sistema de irrigação que levava água do rio às suas plantações. Com a chegada dos europeus e o desvio no fornecimento de água, esses índios sentiram a necessidade de migrar para a zona urbana, sofrendo importantes mudanças socioculturais. A considerável redução da atividade física e as mudanças nos seus hábitos alimentares foram determinantes para franco ganho de peso. Hoje, além de obesa, tal população apresenta a maior prevalência de diabetes melito tipo 2 (DM2) registrada no mundo.

O segundo exemplo de contribuição significativa dos estudos de populações migrantes vem da população japonesa. Neste caso, as distâncias viajadas foram maiores, do Oriente para o Ocidente. Originariamente, a população japonesa caracterizava-se por baixa morbimortalidade por diabetes melito (DM) e doença cardiovascular. Mudanças socioculturais no Ocidente associaram-se a aumento no risco destas doenças nos migrantes japoneses nas Américas. Invariavelmente, a reflexão sobre esse movimento migratório nos remete aos contrastes existentes entre os hábitos alimentares dos habitantes dessas duas regiões do Globo.

Estudos conduzidos há várias décadas nos Estados Unidos foram pioneiros em explorar os efeitos da imigração japonesa a estados como o Havaí, Califórnia e Washington (2-4). O movimento migratório ao Brasil que completou 100 anos - foi posterior ao americano, tendo ocorrido em duas ondas principais, de 1908 a 1941 e de 1953 a 1963. Tal fato permitiu a coexistência de diferentes gerações de nipo-brasileiros no momento presente no Brasil. Atualmente, a maior população nikkey fora do Japão vive em território brasileiro, especialmente no estado de São Paulo (5). Diversos pesquisadores foram atraídos para investigação do impacto do ambiente do Brasil nesses brasileiros "geneticamente" japoneses, entre eles o Japanese-Brazilian Diabetes Study (JBDS) Group (6).

Neste artigo, utiliza-se da experiência do JBDS para compartilhar lições obtidas com a imigração japonesa ao Brasil sob o ângulo da adiposidade corporal. Uma síntese dos achados das diferentes fases deste estudo relacionados ao tema será apresentada.

\section{JBDS}

Em meados dos anos 1980, existiam evidências de que o DM se constituía em importante problema de saúde pública na população japonesa do Brasil. Estudo de mortalidade por DM realizado na cidade de São Paulo revelou que, na primeira geração de nipo-brasileiros (nascidos no Japão ou issei), essa taxa era duas vezes a observada no Japão (7). Em 1987, pesquisadores da Universidade Federal de São Paulo (Unifesp) realizaram os primeiros estudos sobre a prevalência autorreferida de DM2 com comunidades nikkeys do município de São Paulo, ano que se atribuiu a criação do JBDS Group (8). As cifras de DM autorreferida eram semelhantes às descritas no estudo brasileiro de prevalência de DM (9), fundamentado em teste oral de tolerância à glicose (TOTG). Isso era o mesmo que afirmar que os valores estavam subestimados, provavelmente da ordem de $50 \%$. O local selecionado para desenvolver o estudo de base populacional de prevalência de DM foi a cidade de Bauru, SP. 
Da primeira fase (1993) participaram 647 nipobrasileiros (toda a primeira e um terço da segunda geração) entre 40 e 79 anos de idade, entre os quais havia $22,6 \%$ de portadores de DM, sem diferença significante entre os sexos (10). Esta prevalência era três vezes a registrada no Japão e bastante semelhante à dos nipoamericanos residentes em Seattle (11). Era instigante descobrir por que a população nipo-brasileira, exposta ao mesmo ambiente que o restante dos brasileiros, apresentava prevalência tão mais elevada. A atenção voltava-se, então, para os fatores ambientais. Análises preliminares já apontavam associação entre ganho de peso na vida adulta com a presença de distúrbios da tolerância à glicose (12). Aparentemente, a coexistência de gerações permitiria conhecer se a manutenção de costumes mais orientais por parte da primeira geração (issei) teria alguma proteção contra ganho de peso e distúrbios metabólicos. Analisando-se por escalas sociométricas, verificou-se que, de fato, a segunda geração (nisei), em média, afastava-se mais dos hábitos tradicionais japoneses (Figura 1). Apesar disso, as diferenças de prevalências de DM entre as gerações não foram significantes.

Ainda mais alarmante foi a prevalência encontrada na segunda fase do estudo (2000), quando $36 \%$ dos nipo-brasileiros apresentavam DM, uma das maiores taxas detectadas mundialmente (13). Sendo a obesidade o principal fator de risco para essa doença, sua presença foi também investigada, bem como outras doenças frequentemente associadas a distúrbios do metabolismo da glicose. O conjunto dos achados desencadeou investigações de possíveis determinantes do quadro de morbidade dessa população.

Diante do cenário encontrado, o JBDS ampliou seu foco original de investigação, passando a ser denominado Estudo de Diabetes e Doenças Associadas na População Nipo-Brasileira. Sua terceira fase foi um estudo longitudinal, de intervenção nos hábitos de vida, desenvolvida entre 2005 e 2007.

Investigações sobre as interrelações da obesidade sob o aspecto de seus possíveis determinantes causais nos imigrantes japoneses, ou das suas consequências à saúde ou da resposta a programa comunitário de mudanças no estilo de vida - vêm permeando o estudo desde sua origem. Nos tópicos seguintes deste artigo, estes aspectos da adiposidade corporal dos nipo-brasileiros serão abordados.

\section{A ADIPOSIDADE CORPORAL EM NIPO-BRASILEIROS}

O comportamento crescente da obesidade nos países desenvolvidos e em desenvolvimento tem resultado aumento das comorbidades e perda de qualidade de vida. No Brasil, as cifras de excesso de peso nos adultos já ultrapassam, em muito, as de desnutrição (14). Embora existam análises da prevalência em estratos da popula-
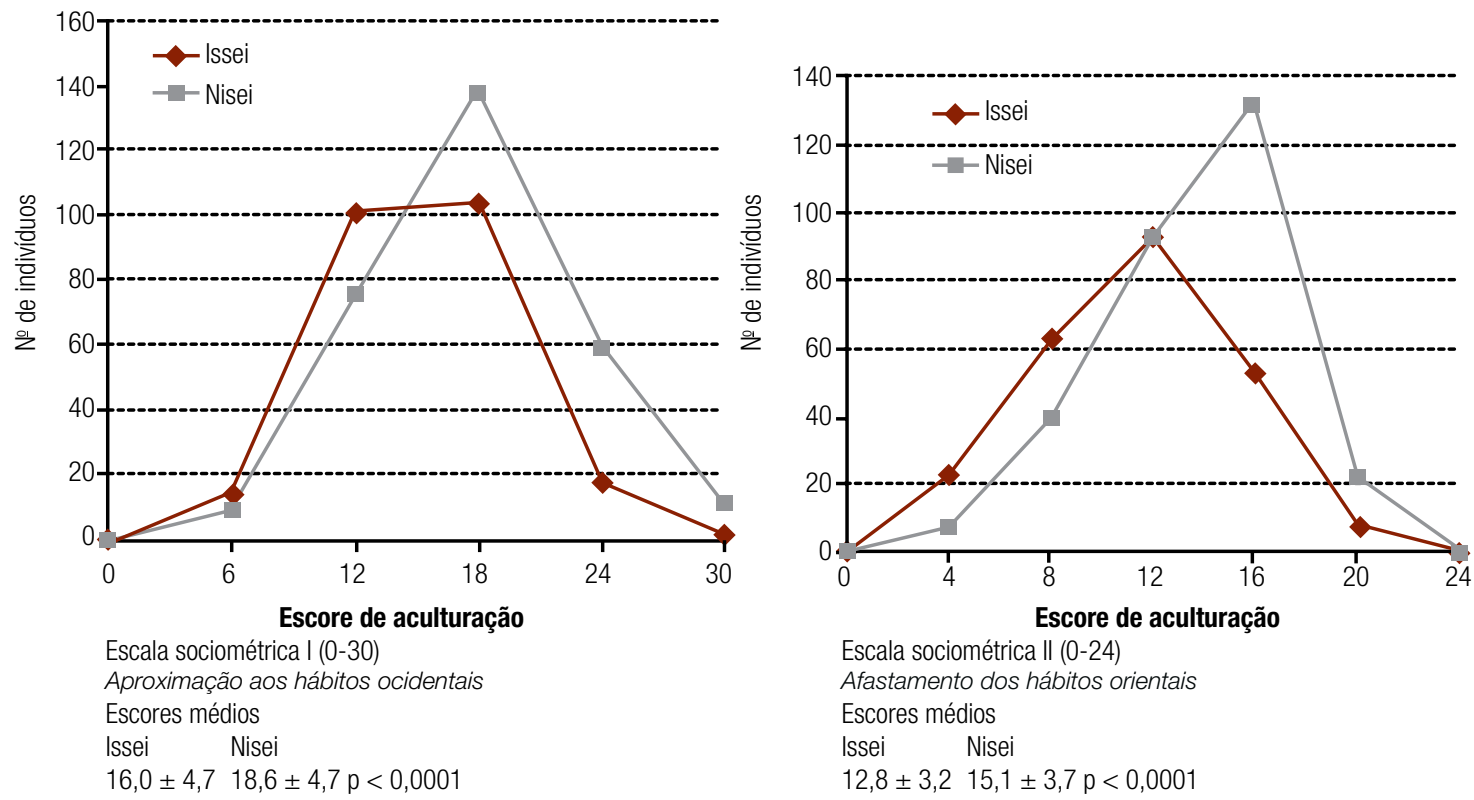

Kikuchi \& lunes. IDF Congress. Helsinki, 1997.

Figura 1. Aculturação de nipo-brasileiros medida por escalas sociométricas segundo a geração. 
ção brasileira quanto a gênero e classe socioeconômica, dados nacionais nos descendentes asiáticos não estão disponíveis. Porém, o JBDS tem relatado dados obtidos nos nipo-brasileiros residentes em Bauru.

Está bem estabelecido que critérios diagnósticos de obesidade devem ser particularizados para as diferentes populações e etnias. Os valores de corte ou são fundamentados em dados estatísticos obtidos da populaçãoreferência ou no excesso de morbimortalidade associado com o aumento do conteúdo de gordura. Asiáticos tendem a ter maior grau de adiposidade corporal para mesmo IMC quando comparados aos caucasoides. Aplicando-se o clássico valor de $30 \mathrm{~kg} / \mathrm{m}^{2}$ para definir obesidade, o National Nutrition Survey do Japão, de 1990-94, identificou menos de 3\% de obesos e cerca de $20 \%$ de portadores de sobrepeso (15). Nos nipobrasileiros, utilizando os mesmos valores de corte, as prevalências observadas foram bem comparáveis às do Japão, conforme mostrado na Tabela 1 (6).

Tabela 1. Prevalência (\%) de sobrepeso e obesidade na população nipo-brasileira de Bauru, segundo sexo e geração, em 1993 e 2000

\begin{tabular}{lccccccccc}
\hline & \multicolumn{4}{c}{ Homens } & \multicolumn{4}{c}{ Mulheres } \\
& \multicolumn{3}{c}{ Sobrepeso } & \multicolumn{2}{c}{ Obesidade } & \multicolumn{2}{c}{ Sobrepeso } & \multicolumn{2}{c}{ Obesidade } \\
& $\mathbf{1 9 9 3}$ & $\mathbf{2 0 0 0}$ & $\mathbf{1 9 9 3}$ & $\mathbf{2 0 0 0}$ & $\mathbf{1 9 9 3}$ & $\mathbf{2 0 0 0}$ & $\mathbf{1 9 9 3}$ & $\mathbf{2 0 0 0}$ \\
\hline $1^{\text {a } \text { geração }}$ & 26,3 & 26,1 & 3,3 & 3,7 & 27,3 & 27,9 & 7,3 & 6,6 \\
$2^{\text {a } \text { geração }}$ & 36,8 & 45,1 & 12,3 & 11,7 & 36,2 & 32,4 & 8,2 & 10,0 \\
\hline
\end{tabular}

O JBDS recalculou essas prevalências aplicando critérios da Japanese Society for the Study of Obesity, que define excesso de peso a partir de $26,4 \mathrm{~kg} / \mathrm{m}^{2}$. Dessa forma, na primeira fase do estudo, $22,4 \%$ dos nipobrasileiros já eram caracterizados como portadores de peso excessivo, em particular os homens de segunda geração (16).

$\mathrm{O}$ encontro de cifras baixas de obesidade em asiáticos usando o clássico valor de corte de $30 \mathrm{~kg} / \mathrm{m}^{2}$ motivou especialistas da Organização Mundial da Saúde (OMS) a rever os critérios e elaborar diretrizes para o diagnóstico, manejo e prevenção de obesidade em países asiáticos (17). Segundo estes, valores acima de 22,9 $\mathrm{kg} / \mathrm{m}^{2}$ diagnosticam obesidade em asiáticos; obesidade central ou abdominal foi definida por circunferências da cintura $\geq 90 \mathrm{~cm} \mathrm{e} \geq 80 \mathrm{~cm}$, para homens e mulheres, respectivamente.

Além da deposição geral de gordura corporal, igualmente importante é o acúmulo de gordura visceral em virtude de seu papel na gênese da resistência à insulina.
Estudos em nipo-americanos apontaram que a área de tecido adiposo visceral, medida por tomografia computadorizada, foi preditiva de distúrbios do metabolismo da glicose (18).

Utilizando critérios específicos para asiáticos, o JBDS estimou as prevalências de obesidade generalizada e central (Tabela 2) confirmando serem estas importantes problemas de saúde entre os nipo-brasileiros, que pode estar contribuindo para as elevadas frequências de DM2 e síndrome metabólica (6).

\begin{tabular}{|c|c|c|c|c|}
\hline & \multicolumn{2}{|c|}{ Homens } & \multicolumn{2}{|c|}{ Mulheres } \\
\hline & 1993 & 2000 & 1993 & 2000 \\
\hline $1^{\mathrm{a}}$ geração & 23,7 & 32,1 & 58,1 & 49,2 \\
\hline $2^{\mathrm{a}}$ geração & 41,8 & 45,3 & 61,8 & 48,5 \\
\hline
\end{tabular}

As controvérsias a respeito dos valores de corte mais adequados para identificar risco em indivíduos de diferentes etnias tiveram repercussões sobre os critérios diagnósticos de síndrome metabólica. Uma análise comparativa das prevalências da síndrome pelos critérios mais frequentemente empregados revelou que, por qualquer deles, mais da metade dos nipo-brasileiros de Bauru apresentam esse diagnóstico (19).

Uma vez detectada essa situação, fez-se necessária a investigação de possíveis fatores causais para o aumento do peso corporal e deposição intra-abdominal da gordura. Nessa linha, foram obtidas informações relativas à atividade física e à dieta destes indivíduos, coletadas por meio de questionários estruturados, aplicados por entrevistadores treinados.

\section{HÁBITOS DE VIDA DOS NIPO-BRASILEIROS}

Aos imigrantes japoneses foi imposto processo de adaptação às condições locais com mudanças marcantes em seus hábitos de vida, sobretudo nos hábitos alimentares, mas também na atividade física. $\mathrm{O}$ padrão alimentar tipicamente japonês foi se mesclando aos hábitos alimentares de outros imigrantes residentes no país e à disponibilidade e acesso a alimentos. Esse processo certamente contribuiu para o quadro atual de morbimortalidade dos nipo-brasileiros de Bauru que difere dos japoneses residentes no Japão. Alimentos frequentemente encontrados na dieta no Japão - soja e seus derivados, peixe e chá verde -, que têm sido associa- 
dos a fatores de proteção contra doença isquêmica do coração, não fazem parte da dieta rotineira do brasileiro. Porém, na atualidade, não obstante estável o valor calórico total consumido pelos adultos japoneses nos últimos 30 anos, o National Nutrition Survey do Japão aponta elevação na proporção da gordura ingerida, em paralelo ao aumento do sobrepeso (20).

$\mathrm{Na}$ busca de possíveis determinantes da elevação da gordura corporal nos imigrantes japoneses e seus descendentes no Brasil, o JBDS partiu da hipótese que o binômio "inatividade física - hábitos alimentares inadequados" teria papel relevante. Por meio de questionário que havia sido empregado para avaliar atividade física em nipo-americanos, constatou-se que o nível de atividades físicas da população nipo-brasileira no trabalho e no lazer era, na grande maioria das vezes, classificado como leve ou moderado, sendo as mulheres mais inativas do que os homens (21). O contraste entre a dieta tradicional japonesa (baixo teor de gorduras, proteínas e açúcares simples e rica em fibras) com as "modernas" dietas ocidentais (altamente calóricas e ricas em gorduras) levou à investigação de associações da adiposidade e doenças metabólicas a componentes alimentares. Alto valor calórico total da dieta e/ou certos padrões de ingestão de nutrientes poderiam predispor a obesidade, resistência à insulina e síndrome metabólica nos nipo-brasileiros.

Resultados da primeira fase mostravam que na comparação das dietas consumidas por indivíduos residentes no Japão, nipo-americanos e nipo-brasileiros contrastavam quanto à ingestão relativa de gordura, que nos dois últimos era praticamente o dobro da do Japão (Tabela 3). Na mesma ocasião, analisou-se se havia associação de hábitos dietéticos com distúrbio da tolerância à glicose, mas a natureza transversal dessa fase do estudo limitava especulações em torno desses achados (22).

Tabela 3. Média do consumo de macronutrientes dos japoneses e migrantes japoneses

\begin{tabular}{lccc}
\hline & $\begin{array}{c}\text { Bauru, } \\
\text { Brasil }\end{array}$ & $\begin{array}{c}\text { Seattle, } \\
\text { Estados Unidos }^{\mathbf{a}}\end{array}$ & Japão $^{\mathbf{b}}$ \\
\hline Idade (anos) & $40-79$ & $45-74$ & $45-69$ \\
Energia (kcal) & 2.970 & 2.137 & 2.016 \\
Carboidrato (\%) & 53,1 & 48,5 & 61,1 \\
Proteína (\%) & 14,5 & 16,5 & 14,8 \\
Gordura (\%) & 32,4 & 32,4 & 16,7 \\
\hline
\end{tabular}

a Tsunehara C, Leonetti DL, Fujimoto WY. Am J Clin Nutr. 1990;52:731-8. ${ }^{\text {} ~ K a w a t e ~ R, ~ Y a m a k i d o ~ M, ~}$ Nishimoto Y, Bennett PH, Hamman RE, Knowler WC. Diabetes Care. 1979;2:161-70. Science and Technology Agency. Natural Resources Investigative Society, eds. Standard table of nutritive values of Japanese foods, supplement. 3rd ed. Tokyo: Japanese Ministry of Finance Printing Office; 1976.
Os estudos iniciais que exploraram possível papel da dieta ocidental para deposição de gordura corporal testaram transversalmente a associação entre consumo de macronutrientes e variáveis antropométricas em análise de regressão múltipla (23). A ingestão de proteínas associou-se ao IMC e à circunferência da cintura, após ajuste para sexo, idade, atividade física e geração. Especulou-se que essa população, geneticamente predisposta à resistência à insulina, quando exposta a ambiente hostil, tenderia a depositar gordura na região visceral e expressar distúrbios metabólicos, em concordância com os achados previamente publicados.

A segunda fase do JBDS permitiu aprofundar tal investigação com a vantagem de ter se formado uma coorte de indivíduos examinados nos dois momentos da pesquisa (1993 e 2000). Foi investigado se dieta dos nipo-brasileiros incluídos em $2000(\mathrm{n}=1330)$ associava-se a morbidades e encontrou-se que o consumo de gorduras totais estava independentemente associado à síndrome metabólica (24). Considerando apenas os indivíduos presentes em ambas as fases do estudo, sem síndrome metabólica em 1993, compararam-se as composições das dietas no ponto de partida entre os nipo-brasileiros que desenvolveram ou não a síndrome em 2000 (25). A circunferência da cintura mostrou-se preditiva desta evolução. Apenas no sexo masculino, o consumo de carnes vermelhas se associou de maneira independente à ocorrência de síndrome metabólica em sete anos de seguimento. Interessantemente, quando se acrescentava ao modelo ajuste para a ingestão de gorduras totais da dieta, tal associação deixava de ser significativa. Isso sugere que o conteúdo de gordura das carnes vermelhas possa estar implicado nessa relação.

O JBDS Group já dispunha de elementos suficientes para orientar um programa de intervenção no estilo de vida da população nipo-brasileira de Bauru. Existiam, também, evidências consistentes na literatura sobre a eficácia de mudanças no estilo de vida na prevenção de DM e de outros distúrbios metabólicos (26-29). Porém, programas de prevenção empregados no Primeiro Mundo podem não ser viáveis em uma realidade como a brasileira.

\section{IMPACTO DE UMA INTERVENÇÃO COMUNITÁRIA NOS NIPO-BRASILEIROS}

Estudos epidemiológicos já forneceram evidências inequívocas da eficácia das mudanças no estilo de vida (hábitos dietéticos e atividade física) sobre a incidência de DM2 $(26,27,29)$. Entretanto, complexa infraestrutura e 
grandes montantes de recursos (financeiros e humanos) empregados nesses estudos não são viáveis à realidade dos serviços de saúde pública de países em desenvolvimento. O desafio atualmente é instituir estratégias de intervenção em mudança de estilo de vida adaptadas para a realidade de países em desenvolvimento que também sejam capazes de trazer benefícios em perfil cardiometabólico de populações de risco cardiovascular (30).

Diante do preocupante quadro de morbidade encontrado nessa população nipo-brasileira residente em Bauru, o JBDS Group iniciou a terceira fase do estudo intitulada "Intervenção sobre o estilo de vida da população nipo-brasileira de alto risco para síndrome metabólica, residente em Bauru, SP". A hipótese era a de que um programa de intervenção no estilo de vida resultaria benefícios sobre o perfil de risco cardiovascular de toda a população envolvida.

Em 2005, 728 nipo-brasileiros de primeira e segunda gerações, que haviam participado da segunda fase do estudo, aderiram ao programa de dois anos de intervenção de mudança em estilo de vida. Resultados referentes a 650 indivíduos reavaliados após o primeiro ano de intervenção são aqui mostrados, enquanto os relativos ao segundo ano encontram-se sob análise.

A estratégia de intervenção no estilo de vida baseouse fundamentalmente na orientação de dieta saudável e prática de atividade física $(27,31)$. Os participantes foram agendados para consulta individual com nutricionista, duas sessões em grupo para recomendações de dieta e atividade física e dois eventos de exercício para a comunidade (incluindo caminhada, alongamento e dança) durante o primeiro ano do programa de intervenção. As dietas foram orientadas de acordo com o status nutricional inicial com a seguinte distribuição de ingestão diária: $50 \%$ a $60 \%$ das calorias totais provenientes de carboidratos, $<30 \%$ de gordura total, $<10 \%$ de ácidos graxos saturados e $10 \%$ a $15 \%$ de proteínas, < $300 \mathrm{mg}$ de colesterol e $\geq 15 \mathrm{~g} /$ dia de fibras. No que diz respeito à atividade física, foi orientada a prática de 30 minutos/dia, na maioria dos dias da semana, somando o total de, no mínimo, 150 minutos/semana (31). $\mathrm{O}$ estudo incluiu três momentos de avaliação clínicolaboratorial: basal, após o primeiro ano de intervenção e ao final do segundo ano. Nessas ocasiões, os indivíduos foram submetidos a exame médico, avaliação com nutricionista e educador físico e coletas de sangue para determinação de parâmetros bioquímicos, hormonais e de marcadores inflamatórios. As metas foram: redução de, pelo menos, $5 \%$ do peso, redução do consumo de gordura e aumento de fibras e prática de 30 minutos/ dia na maioria dos dias da semana.

Em 2005, considerando o valor médio de IMC de $24,7 \mathrm{~kg} / \mathrm{m}^{2}$, os participantes estavam acima do peso ideal e apresentavam média de circunferência de cintura elevada $(92,5 \mathrm{~cm}$ para homens e $85,3 \mathrm{~cm}$ para mulheres). Após um ano de intervenção foi observada redução significativa no IMC e na circunferência de cintura. Apesar de redução discreta nos parâmetros antropométricos, houve redução significativa em pressão arterial, glicemia e colesterol total e frações (dados não publicados). Essas reduções foram padronizadas e estão apresentadas na Tabela 4. Os achados do presente estudo são compatíveis com os de outros estudos de intervenção. $\mathrm{O}$ Finnish Diabetes Prevention Study e o Diabetes Prevention Program (DPP) mostraram que é possível a prevenção de DM2 com pequena redução da adiposidade $(27,29)$. Na versão indiana do DPP, o IDPP, houve redução de $26,4 \%$ no risco relativo de desenvolver DM sem alteração significativa de peso ou circunferência de cintura na população (32). Em outro estudo conduzido no Japão, a redução no risco de desenvolver DM foi mais acentuada do que aquela esperada pela diminuição no IMC (33), sugerindo que o efeito benéfico das intervenções pode não ser atribuído somente às reduções na adiposidade corporal (Tabela 5).

Além da análise dos resultados após o segundo ano de intervenção (em andamento), acompanhamento mais longo é necessário para avaliar a persistência dos benefícios vistos após o primeiro ano de intervenção e investigar o impacto desses benefícios na prevenção de DM e eventos cardiovasculares.

\section{CONSIDERAÇÕES FINAIS}

Após 15 anos de trabalho com a população nipo-brasileira de Bauru, reforçou-se a importância de ambos, fatores ambientais e fatores genéticos, para o acúmulo de adiposidade, especialmente na região abdominal, que, por sua vez, é geradora do preocupante perfil de risco cardiovascular desses indivíduos. O ambiente ocidental deletério é fortemente sugerido, considerando-se no país de origem as prevalências de doenças como o DM2, que ocorre com frequência bem menor. Porém, brasileiros não de origem japonesa, expostos a esse mesmo ambiente, desenvolvem DM e dislipidemias com frequências bem inferiores. Dessa forma, pressupõe-se que fatores genéticos também contribuam para esse quadro nos imigrantes japoneses e seus descendentes no Brasil. 


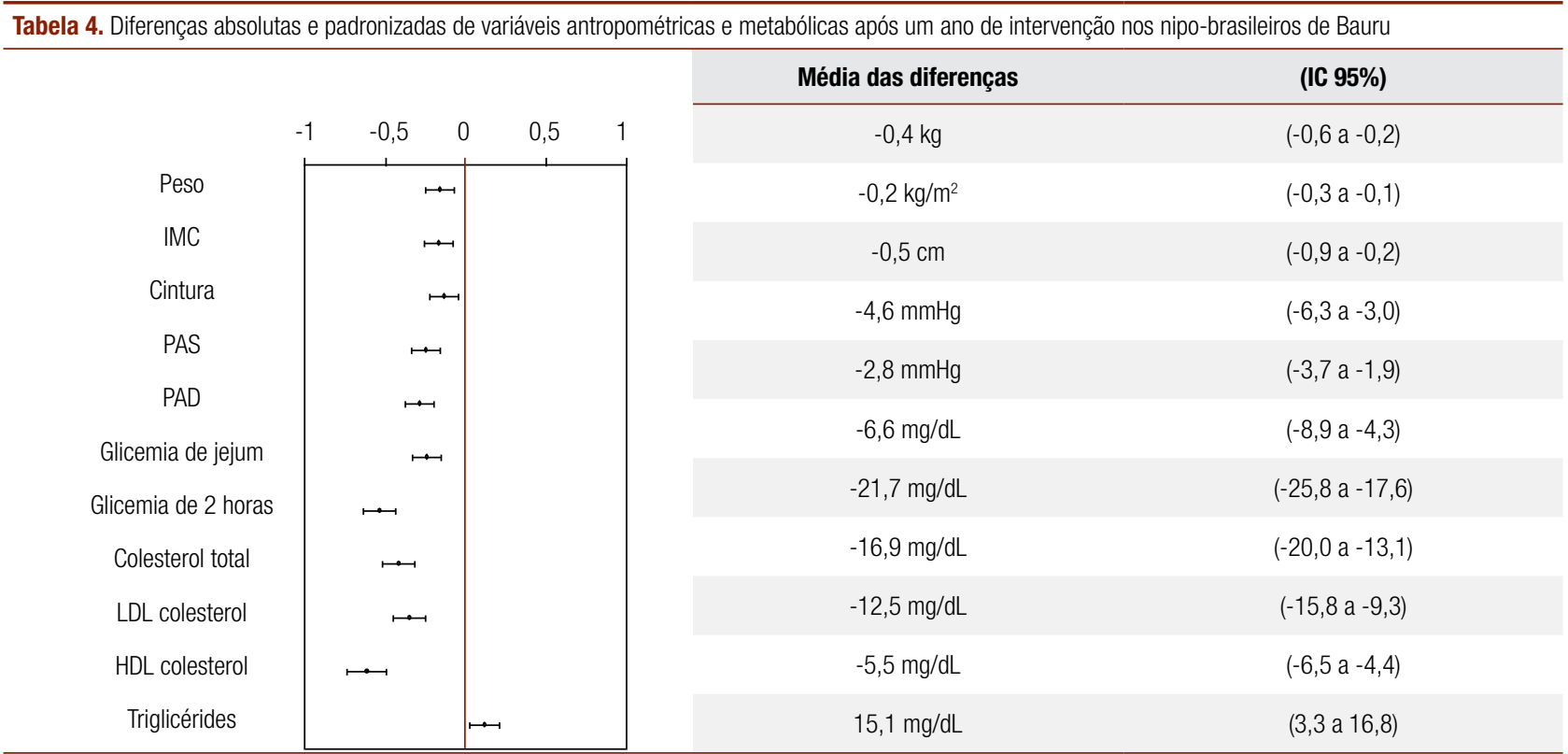

IMC = índice de massa corporal; PAS = pressão arterial sistólica; PAD = pressão arterial diastólica. Diferenças padronizadas = média da diferença da variável/desvio-padrão da mesma variável.

\begin{tabular}{|c|c|c|c|}
\hline & 2000 & 2005 & 2006 \\
\hline Idade (anos) & $56,0(11,2)$ & $61,1(11,2)$ & $61,8(11,1)$ \\
\hline GJA ou TGD/DM (\%) & $58,8 / 37,3$ & $58,4 / 30,1$ & $35,4 / 21,7$ \\
\hline Peso (kg) & $61,5(12,4)$ & $61,6(12,4)$ & $61,2(12,7)^{\#}$ \\
\hline Índice de massa corporal $\left(\mathrm{kg} / \mathrm{m}^{2}\right)$ & $24,8(3,7)$ & $24,9(4,1)^{\star}$ & $24,7(4,1)^{\#}$ \\
\hline Circunferência de cintura (cm) & $84,0(10,1)$ & $88,5(10,4)^{*}$ & $88,1(10,6)^{\#}$ \\
\hline Pressão arterial sistólica (mmHg) & $134,6(25,6)$ & $139,3(21,8)^{\star}$ & $134,3(21,4)^{\#}$ \\
\hline Pressão arterial diastólica (mmHg) & $78,1(13,9)$ & $81,2(12,0)^{*}$ & $78,4(11,6)^{\#}$ \\
\hline Glicemia de jejum (mg/dL) & $110,9(30,9)$ & $112,7(30,9)$ & $105,4(27,3)^{\#}$ \\
\hline Glicemia de 2 horas (mg/dL) & $167,3(79,8)$ & $150,9(52,7)$ & $130,9(45,4)^{\#}$ \\
\hline Colesterol total (mg/dL) & $186,7(36,7)$ & $183,3(36,7)$ & $170,0(33,3)^{\#}$ \\
\hline LDL-colesterol (mg/dL) & $110,0(30,0)$ & $110,0(33,3)$ & $100,0(26,7)^{\#}$ \\
\hline HDL-colesterol (mg/dL) & $43,3(10,0)$ & $46,7(10,0)^{*}$ & $43,3(10,0)^{\#}$ \\
\hline Triglicérides (mg/dL) & $230,3(2,3)$ & $159,4(124,0)^{*}$ & $177,4(141,7)$ \\
\hline
\end{tabular}

GJA = glicemia de jejum alterada; TGD = tolerância à glicose diminuída; $\mathrm{DM}=$ diabetes melito. ${ }^{*} \mathrm{p}<0,05$ para comparações entre 2000 e 2005; \#p $<0,05$ para comparações entre 2005 e 2006 . Valores expressos em média (desvio-padrão), exceto quando indicado.

A terceira fase do JBDS forneceu resultados animadores quanto ao perfil de risco cardiometabólico da população. As medidas antropométricas constataram redução da adiposidade corporal e as dosagens bioquímicas comprovaram melhora do perfil de risco cardiometabólico. A intervenção apresenta a peculiaridade de ter sido fundamentada em baixo montante de recursos para sua implantação. Porém, é importante assinalar que as conclusões são válidas para esse estrato da população brasileira, não podendo extrapolar tais resultados para a população geral.
Desconhece-se se os benefícios alcançados nessa população serão duradouros e se, em longo prazo, reverterão, de fato, em menor incidência de eventos cardiovasculares.

Agradecimentos: Pesquisa financiada pelo Conselho Nacional de Desenvolvimento Científico e Tecnológico (CNPq) (505845/2004-0) e pela Fundação de Amparo à Pesquisa do Estado de São Paulo (Fapesp) (05/50178-7).

Declaração: Os autores declaram não haver conflitos de interesse científico neste artigo. 


\section{REFERÊNCIAS}

1. Knowler WC, Bennett PH, Hamman RF, Miller M. Diabetes incidence and prevalence in Pima Indians: a 19-fold greater incidence than in Rochester, Minnesota. Am J Epidemiol. 1978;108:497-505.

2. Marmot MG, Syme SL, Kagan A, Kato H, Cohen JB, Belsky J. Epidemiologic studies of coronary heart disease and stroke in Japanese men living in Japan, Hawaii and California: prevalence of coronary and hypertensive heart disease and associated risk factors. Am J Epidemiol. 1975;102:514-25.

3. Kawate R, Yamakido M, Nishimoto Y, Bennett PH, Hamman RF, Knowler WC. Diabetes mellitus and its vascular complications in Japanese migrants of the island of Hawaii. Diabetes Care. 1979;2:161-70.

4. Fujimoto WY, Leonetti DL, Kinyoun JL, Shuman WP, Stolov WC, Wahl PW. Prevalence of complications among second-generation Japanese-American men with diabetes, impaired glucose tolerance or normal glucose tolerance. Diabetes. 1987;36:730-9.

5. Centro de Estudos Nipo-Brasileiros. Pesquisa da população de descendentes de japoneses residentes no Brasil. São Paulo: Centro de Estudos Nipo-Brasileiros; 1988.

6. Grupo de Estudos de Diabetes na Comunidade Nipo-Brasileira. Departamento de Medicina Preventiva - UNIFESP e Centro de Estudos Nipo-Brasileiro. "Diabetes mellitus e doenças associadas em nipo-brasileiros". São Paulo: Green Forest do Brasil; 2004.

7. Gotlieb SLD. Mortality among Japanese migrants living in Sao Paulo City. J Public Health. 1990;24:453-67.

8. lunes M, Franco LJ, Wakisaka K, loshida LC, Osiro K, Hirai AT, et al. Self-reported prevalence of diabetes mellitus in 1st (Issei) and 2nd (Nisei) generation of Japanese-Brazilians over 40 years of age. Diabetes Res Clin Pract. 1994;24 Suppl:S53-7.

9. Malerbi DA, Franco LJ. Multicenter study of the prevalence of diabetes mellitus and impaired glucose tolerance in urban Brazilian population aged 30-69 years. Diabetes Care. 1992;15:1509-16.

10. Ferreira SRG, lunes $M$, Franco LJ, lochida LC, Hirai A, Vivolo MA for the Japanese-Brazilian Diabetes Study Group. Disturbances of glucose and lipid metabolism in first and second generation Japanese-Brazilians. Diabetes Res Clin Pract. 1996;34 Suppl:S59-63.

11. Fujimoto WY. The growing prevalence of non-insulin-dependent diabetes in migrant Asian populations and its implications for Asia. Diabetes Res Clin Pract. 1992;15:167-84.

12. Gimeno SGA, Ferreira SRG, Cardoso MA, Franco LJ, lunes M. Weight gain and risk of developing glucose tolerance disturbance: a study of a Japanese-Brazilian population. J Epidemiol. 2000;10(2):103-10.

13. Gimeno SGA, Ferreira SRG, Franco LJ, lunes M. Comparison of glucose tolerance categories according to World Health Organization and American Diabetes Association diagnostic criteria in a population-based study in Brazil. Diabetes Care. 1998;21(11):1889-92.

14. Instituto Brasileiro de Geografia e Estatística (IBGE). Pesquisa de orçamentos familiares, 2002-2003 (POF): análise da disponibilidade domiciliar de alimentos e do estado nutricional no Brasil. Rio de Janeiro; 2004.

15. Yoshiike N, Matsumura Y, Zaman MM, Yamaguchi M. Descriptive epidemiology of body mass index in Japanese adults in a representative sample from the National Nutrition Survey 1990-94. Int J Obes. 1998;22:684-7.

16. Lerario DDG, Gimeno SGA, Franco LJ, Ferreira SRG. Excesso de peso e implicações da gordura abdominal para a síndrome metabólica em nipo-brasileiros. Rev Saude Publica. 2002;36(1):4-11.
17. Inoue S, Zimmet P. The Asia-Pacific perspective: redefining obesity and its treatment. WHO Western Pacific Region. Australia; 2000. World Health Organization (WHO) 2000. Steering Committee of the Western Pacific Region of the WHO, the International Association for the Study of Obesity Task Force. The Asia-Pacific perspective: redefining obesity and its treatment.

18. Bouyko EJ, Leonetti DL, Fujimoto WY, Newell-Morris L. Visceral adiposity and risk of type 2 diabetes. Diabetes Care. 2000;23:465-71.

19. Rosenbaum P, Gimeno SGA, Sanudo A, Franco LJ, Ferreira SRG. Analysis of criteria for metabolic syndrome in a population-based study of Japanese-Brazilians. Diabetes Obes Metab. 2005;7(4):352-9.

20. Yoshiike N, Matsumura $Y$, Iwaya M, Sugiyama M, Yamaguchi M. National Nutrition Survey in Japan. J Epidemiol. 1996;6:S189-200.

21. Doro AR, Gimeno SGA, Hirai AT, Matsumura LK, Ferreira SRG. Análise da associação de atividade física à síndrome metabólica em estudo populacional de nipo-brasileiros. Arq Bras Endocrinol Metab. 2006;50(6):1066-74.

22. Costa MB, Ferreira SRG, Franco LJ, Gimeno SGA, lunes M for the JBDS Group. Dietary patterns in an at risk population for glucose intolerance. J Epidemiol. 2000;10(2):111-7.

23. Ferreira SRG, Lerario DDG, Gimeno SGA, Sanudo A, Franco LJ for the Japanese-Brazilian Diabetes Study Group. Obesity and central adiposity in Japanese immigrants: role of the Western dietary pattern. J Epidemiol. 2002;12:431-8.

24. Freire RD, Shinzato AR, Cardoso MA, Ferreira SRG for the JBDSG. Dietary fat is associated with metabolic syndrome in JapaneseBrazilians. Diabetes Care. 2005;28(7):1779-85.

25. Freire RD, Castro TG, Cardoso MA, Gimeno SGA, Ferreira SRG. Dietary intakes associated with metabolic syndrome in a cohort of Japanese ancestry. Br J Nutr. 2006;96(3):532-8.

26. Pan XR, Li GW, Hu UH. Effect of diet and exercise in preventing DM type 2 in people with impaired glucose tolerance. The Da Qing IGT and Diabetes Study. Diabetes Care. 1997;20:537-54.

27. Tuomilehto J, Lindstrom J, Eriksson JG, Valle TT, Hamalainen $\mathrm{H}$, llanne-Parikka $P$, et al. Prevention of type 2 dabetes mellitus by changes in lifestyle among subjects with impaired glucose tolerance. N Engl J Med. 2001;344(18):1343-50.

28. Ilanne-Parikka P, Eriksson JG, Lindström J, Peltonen M, Aunola S, Hämäläinen $\mathrm{H}$, et al. Effect of lifestyle intervention on the occurrence of metabolic syndrome and its components in the Finnish Diabetes Prevention Study. Diabetes Care. 2008;31:805-7.

29. Knowler WC, Barrett-Connor E, Fowler SE, Hamman RF, Lachin JM, Walker EA, et al. The Diabetes Prevention Program: reduction in the incidence of type 2 diabetes with lifestyle intervention or metformin. N Engl J Med. 2002;346:393-403.

30. Ferreira SRG, Almeida B, Siqueira AFA, Khawali C. Intervenções na prevenção do diabetes mellitus tipo 2: é viável um programa populacional no nosso meio? Arq Bras Endocrinol Metab. 2005;49(4):479-84.

31. World Health Organization \& Food and Agricultural Organization. The scientific basis for diet, nutrition and the prevention of type 2 diabetes. Geneva; 2003.

32. Ramachandran A, Snehalatha C, Mary S, Mukesh B, Bhaskar AD, Vijay V, et al. The Indian Diabetes Prevention Programme shows that lifestyle modification and metformin prevent type 2 diabetes in Asian Indian subjects with impaired glucose tolerance (IDPP-1). Diabetologia. 2006;49(2):289-97.

33. Kosaka K, Noda M, Kuzuva Y. Prevention of type 2 diabetes by lifestyle intervention: a Japanese trial in IGT males. Diabetes Res Clin Pract. 2005;67(2):152-62. 